\title{
The Superslow Pulsation X-ray Pulsars in High Mass $\mathrm{X}$-ray Binaries
}

\section{Wei Wang*}

National Astronomical Observatories, Chinese Academy of Sciences, Beijing 100012, China

E-mail: wangweidbao.ac.cn

There exists a special class of X-ray pulsars with a very slow pulsation with $P_{\text {spin }}>>1000 \mathrm{~s}$ in the high mass X-ray binaries (HMXBs). Presently two superslow pulsation X-ray pulsars are discovered, 4U 2206+54 with $P_{\text {spin }} \sim 5560 \mathrm{~s}$ and $2 \mathrm{~S} 01114+65$ with $P_{\text {spin }} \sim 9600 \mathrm{~s}$. Longterm monitoring observations find spin period evolution of two sources: spin-down trend for 4U $2206+54\left(P_{\text {spin }}^{\cdot} \sim 4.9 \times 10^{-7} \mathrm{~s} \mathrm{~s}^{-1}\right)$ and long-term spin-up trend for $2 \mathrm{~S} 0114+65\left(P_{\text {spin }}^{\cdot} \sim\right.$ $\left.-1 \times 10^{-6} \mathrm{~s} \mathrm{~s}^{-1}\right)$ in the last 20 years. Additionally, spectral properties of two superslow pulsation $\mathrm{X}$-ray pulsars show the similar behaviours in X-rays: lower hydrogen column density and harder spectrum at the flux maximum. This characteristics suggested that both two objects belong to the highly obscured binary systems similar to some other supergiant X-ray binaries. The hard X-ray tails above $70 \mathrm{keV}$ are discovered in 2S 0114+65 implying the possible accretion processes similar to a black hole system with the existence of possible hot corona or transient jet. The cyclotron absorption lines at $\sim 30 \mathrm{keV}$ and $60 \mathrm{keV}$ in the spectrum of $4 \mathrm{U} 2206+54$ suggest a magnetized neutron star with $B \sim 3 \times 10^{12} \mathrm{G}$. The physical origin of the superslow spin period is still unclear. The possible origin and evolution channel of the superslow pulsation X-ray pulsars are briefly discussed. We suggested that the superslow pulsation X-ray pulsars should be the young neutron star accreting binaries.

The Extreme and Variable High Energy Sky - extremesky2011,

September 19-23, 2011

Chia Laguna (Cagliari), Italy

\footnotetext{
* Speaker.
} 


\section{Introduction}

Spin period of neutron stars in the Universe is observed in a wider range in various systems: $1.3 \mathrm{~ms}-10 \mathrm{~s}$ for radio pulsars; $2-12 \mathrm{~s}$ for magnetars; $1.5-30 \mathrm{~ms}$ for millisecond pulsars in low mass X-ray binaries; $0.1-\sim 1000 \mathrm{~s}$ for Be X-ray transients; $10-\sim 1000 \mathrm{~s}$ for supergiant $\mathrm{X}$-ray binaries. There still exist the neutron star systems with a much longer spin period. Two possible superslow X-ray pulsar candidates were reported recently: 1E 161348-5055 in a young

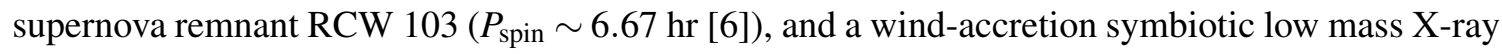
binary 4U 1954+319 ( $P_{\text {spin }} \sim 5 \mathrm{hr}[\mathbb{[ 1 6 ]})$. But the observed modulations possibly due to an orbital period cannot be excluded. The other two superslow X-ray pulsars are discovered in HMXBs: $4 \mathrm{U}$

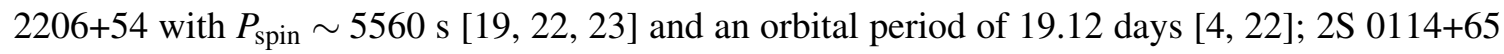
with $P_{\text {spin }} \sim 9600$ s [ [8, 24] and an orbital period of 11.59 days [[]].

Two superslow X-ray pulsars are powered by the direct wind-fed accretion from the stellar winds of the companion stars. But they have different types of the companions. The optical counterpart of $4 \mathrm{U} 2206+54$ at a distance of $\sim 2.6 \mathrm{kpc}$ was identified as an $09.5 \mathrm{~V}$ star with a high He abundance[[]]. For 2S 0114+65, a type B1a supergiant optical counterpart (LS I+65010) at a distance of $\sim 7.2 \mathrm{kpc}$ was identified [ए8].

In this paper, we will probe the spin period evolution of two superslow pulsation pulsars, and spectral properties, specially orbital phase-resolved spectra of two sources with INTEGRAL longterm monitoring observations. These studies will help us to understand the physical origin and evolution of this peculiar class of neutron star binaries.

\section{Spin period variations of superslow pulsation X-ray pulsars}

\section{$2.14 \mathrm{U} 2206+54$}

The spin period of the neutron star in 4U 2206+54 was discovered in 2009 [10, 22]. Reig et al. [ए]] found the spin period of 5559 $\pm 3 \mathrm{~s}$ around MJD 54240 using RXTE/PCA data; and obtained a spin period of 5525 $\pm 30 \mathrm{~s}$ using EXOSAT observations around 1984. Wang [22], [23] found a spin period of $\sim 5550 \pm 50$ around MJD 53719 using INTEGRAL/IBIS data. Finger et al. [Q] obtained a spin period of $5554 \pm 9 \mathrm{~s}$ at MJD 54237 using the Suzaku data; and they also found a spin-period of $5420 \pm 28 \mathrm{~s}$ around MJD 51141 by re-analyzing the BeppoSAX data, suggesting a spin-down of neutron star in $4 \mathrm{U} 2206+54$ with a spin-down rate of $\sim(5.0 \pm 1.3) \times 10^{-7} \mathrm{~s} \mathrm{~s}^{-1}$ when they compared the results by Suzaku and BeppoSAX. With the long-term INTEGRAL observations, we[25] determined the spin period at $5559.3 \pm 1.9 \mathrm{~s}$ in MJD 54087 and $5566.7 \pm 7.0 \mathrm{~s}$ in MJD 54258 deriving a spin-down rate of $(4.9 \pm 3.2) \times 10^{-7} \mathrm{~s} \mathrm{~s}^{-1}$ from MJD $54080-54260$ [25]].

\section{$2.22 \mathrm{~S} \mathrm{0114+65}$}

The X-ray source $2 \mathrm{~S} 0114+65$ consists of an wind-fed accretion neutron star with its pulse period first measured at $\sim 2.78 \mathrm{hr}[\mathbb{[ 1 0}]$. Hall et al.[ए]] derived a period of $\sim 2.73 \mathrm{hr}$, and found a spin-up rate of $\sim 6.2 \times 10^{-7} \mathrm{~s} \mathrm{~s}^{-1}$ for the neutron star in $2 \mathrm{~S} 0114+65$ over $\sim 11 \mathrm{yr}$. Bonning \& Falanga[B]] obtained a spin period of $\sim 2.67 \mathrm{hr}$, and a spin-up rate of $\sim 8.9 \times 10^{-7} \mathrm{~s} \mathrm{~s}^{-1}$ from 1996 to 2004. Farrell et al.[[8] analyzed RXTE data around early 2006 to derive a spin period of $\sim 2.65 \mathrm{hr}$. With the INTEGRAL long-term monitoring observations, Wang[24] found a pulse 

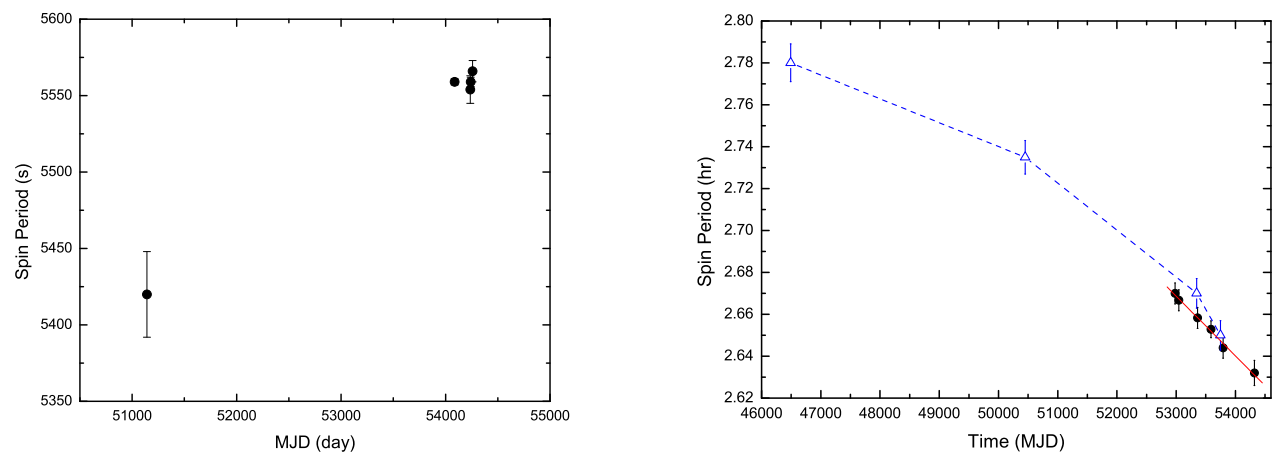

Figure 1: Spin evolution history of 4U 2206+54 (left) and 2S 0114+65 (right) from different observations.

period evolution of $\sim 2.67$ hour to 2.63 hour from 2003 - 2008 and derived a spin-up rate of $1.09 \times 10^{-6} \mathrm{~s} \mathrm{~s}^{-1}$. Compared with the previous reported spin-up rate, the spin-up rate of the neutron star in $2 \mathrm{~S} 0114+65$ is accelerating (see Fig. 1).

\section{Spectral properties of superslow pulsation X-ray pulsars}

Both superslow pulsation X-ray pulsars are highly variable X-ray sources, with a luminosity range of $10^{34}-10^{36} \mathrm{erg} \mathrm{s}^{-1}$. We present the orbital phase-resolved spectral properties for $4 \mathrm{U} 2206+54$ and $2 \mathrm{~S} 0114+65$ in Figs. $2 \& 3$ respectively. The spectra are fitted with the absorbed power-law model plus high energy cut-off. The spectral variations in both two sources show a common property. There exist anti-correlations between the flux and hydrogen column density/photon index, i.e., a lower column density and harder spectrum around maximum of X-ray flux. Orbital phase modulation of the hydrogen column density has been also detected in other high mass X-ray binaries like Vela X-1 [2] and EXO 1722-363 [20]. These spectral behaviour over the orbital phase suggested that they should belong to highly obscured X-ray binary systems.

We also search for the possible cyclotron line features in hard X-ray spectra in two systems. The cyclotron absorption lines at $\sim 30 \mathrm{keV}$ and $60 \mathrm{keV}^{1}$ are detected in $4 \mathrm{U} 2206+54$ (Fig. 4, see [22], [25]), suggesting a magnetized neutron star with the magnetic field of $\sim 3 \times 10^{12} \mathrm{G}$ located in the binary. Interestingly, we find that the cyclotron line features of $4 \mathrm{U} 2206+54$ are only detected in a small part (25\%) of the whole orbital phase (just before the peak flux)[R5]. Unfortunately, we have not find evidence for the magnetic neutron star in 2S 0114+65 with different observations.

In addition, a high energy tail was discovered in the X-ray spectrum of $2 \mathrm{~S} 0114+65$ [Q, [24]. With detailed studies using INTEGRAL data, we find that high column density may lead to the disappearance of the hard X-ray tails in the spectra[24]: when the derived values of column density are higher than $\sim 3 \times 10^{22} \mathrm{~cm}^{-2}$, no hard X-ray tails are detected. How to produce the hard X-ray tails above $70 \mathrm{keV}$ for accreting neutron star in high mass $\mathrm{X}$-ray binaries especially in the wind-fed accretion systems is unclear. It is possible that hot corona exists near neutron stars for wind-fed

\footnotetext{
${ }^{1}$ Some residuals in the INTEGRAL data around $60 \mathrm{keV}$ may be expected due to calibration and background uncertainties. This absorption feature need further observations by other missions.
} 


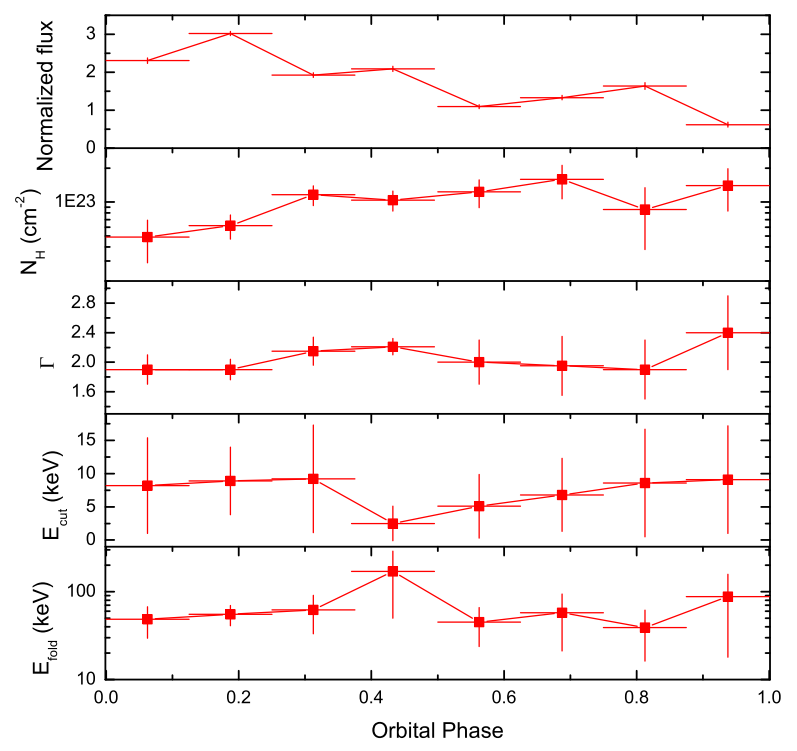

Figure 2: Spectral property variations [25] of $4 \mathrm{U} 2206+54$ with orbital phases.
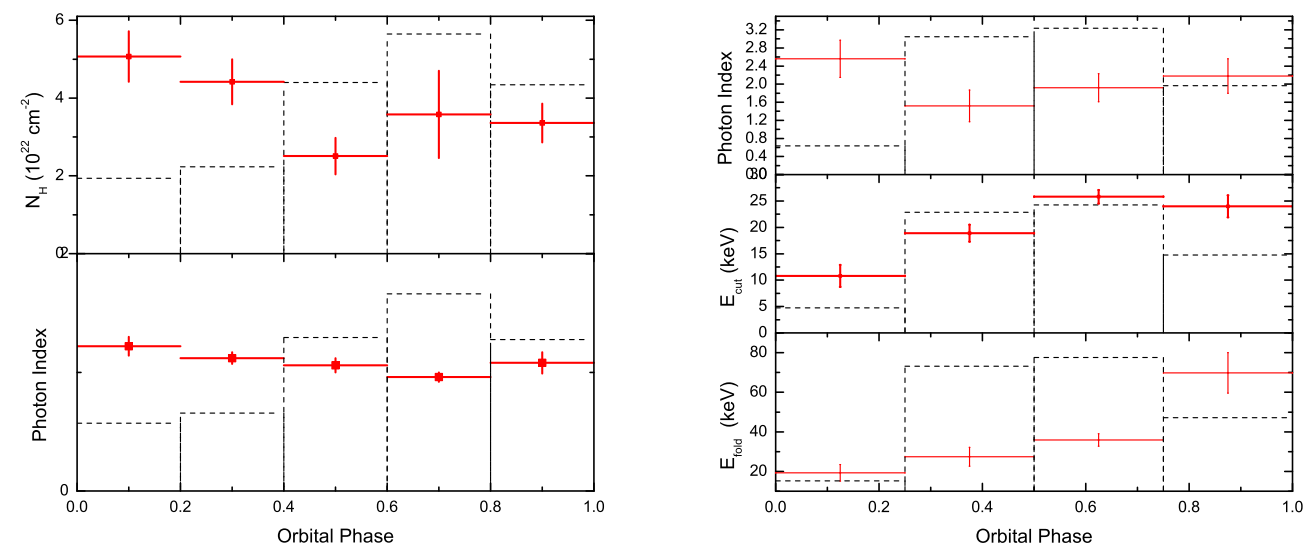

Figure 3: Spectral property variations of $2 \mathrm{~S} 0114+65$ with orbital phases (left from RTXE [8]; right from INTEGRAL [24]). The dashed lines show the normalized flux variations.

accretion systems like $2 \mathrm{~S} 014+65$; and the dense accretion materials or strong winds prevent the formation of hot corona or depress the comptonization effects.

\section{Possible origin and evolution channels of superslow pulsation X-ray pulsars}

The spin period evolution of the new-born neutron star generally undergoes three states [四]: $e$ jector state in which neutron star spins down through the conventional spin-powered pulsar energyloss mechanisms; propeller state in which spin period decreases by means of interaction between the neutron star magnetosphere and stellar wind of the companion; accretor state in which spin 


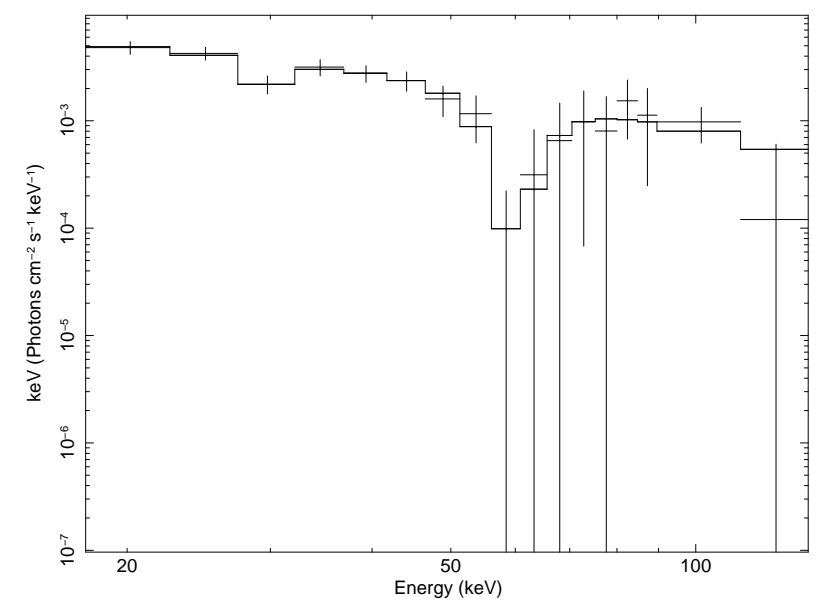

Figure 4: The X-ray spectrum of $4 \mathrm{U} 2206+54$ with two cyclotron absorption lines at $\sim 30 \mathrm{keV}$ and 60 $\mathrm{keV}[22]$.

period of neutron star reaches a critical value, and the neutron star begins to accrete materials on to the surface, then switches on as the X-ray pulsar. The critical period is defined by equating the corotational radius of the neutron star to the magnetospheric radius, which induces the longest period of several hundred seconds but less than $\sim 1000 \mathrm{~s}$ for the neutron star of magnetic field $B<B_{\text {cr }}=4.4 \times 10^{13} \mathrm{G}$. Then what is the formation channel for the superlong spin period $>>1000$ $\mathrm{s}$ in this evolutional track?

Li \& van den Heuvel [ㄷ] have suggested that neutron star spins down to the spin period range longer than $1000 \mathrm{~s}$ if the neutron star was born as a magnetar with an initial magnetic field $\geq 10^{14} \mathrm{G}$. This ultra-strong magnetic field could decay to the normal value ranges of $10^{12}-10^{13}$ $\mathrm{G}$ within a few million years, so that $4 \mathrm{U} 2206+54$ and $2 \mathrm{~S} 0114+65$ may be defined as magnetar descendants. The alternative suggestion[[13] shows that an additional evolution phase subsonic propeller state between the transition from supersonic propeller state to accretor state could allow the spin period increases up to several thousand seconds without the assumption of magnetars. The superslow pulsation neutron star could be formed by the quasi-spherical accretion in windfed accreting systems, which is applied to magnetized neutron stars in symbiotic X-ray binaries [ए7]. Finger et al. [9] interpreted that the neutron star in $4 \mathrm{U} 2206+54$ should be a magnetar with a magnetic field of $\sim(3-5) \times 10^{15} \mathrm{G}$. As we know, magnetar activity would emit X-rays with a typical luminosity of $\sim 10^{35}-10^{36} \mathrm{erg} \mathrm{s}^{-1}$ and a typical power-law spectrum in hard X-ray bands $(10-100 \mathrm{keV})$ of photon index $\Gamma \sim 1-1.5$ for known magnetar candidates [114, 2]], which is inconsistent with the observed properties of $4 \mathrm{U} 2206+54$. Magnetar assumption must make present magnetar X-ray luminosity lower than $10^{34} \mathrm{erg} \mathrm{s}^{-1}$ for the case of $4 \mathrm{U} 2206+54$. It is still quite interesting that the discovery of the cyclotron absorption line feature around $30 \mathrm{keV}$ would suggest a magnetic field of $3 \times 10^{12} \mathrm{G}$ for the electron cyclotron absorption case, but a magnetic field of $\sim 5 \times 10^{15} \mathrm{G}$ for the proton cyclotron line assumption. Thus, difficulty and uncertainties in explaining the long spin period still exist. It is possible that the X-ray pulsar in $4 \mathrm{U} 2206+54$ may not follow the present standard evolution models in close binaries.

Finally, a possible evolution track for the slow-pulsation neutron stars in wind-fed accreting binaries is suggested. There may exist the physical link among slow-pulsation X-ray pulsars: $4 \mathrm{U}$ 
$2206+54$ and $2 S 0114+65$ are the younger systems undergoing rapid spin period evolutions, from rapid spin-down of $4 \mathrm{U} 2206+54$ to rapid spin-up of 2S 0114+65; other supergiant X-ray binaries, like Vela X-1 and EXO 1722-363 are the older systems in the equilibrium spin period range after the rapid evolution phase. In the equilibrium, accreting X-ray pulsars in wind-fed systems would not undergo long-term spin-up/down trends, but the torque changes and fluctuations occur during accretion. This evolution track assumption requires further long-term observations: the spin-up/down process continues in a longer time scale or there exist torque changes in future. These important issues will help us to well understand the origin and evolution of superslow pulsation neutron star systems, and provide new information on the evolution channels of accreting neutron star high mass X-ray binaries.

\section{References}

[1] Bhattacharya, D. \& van den Heuvel, E.P.J., 1991, Phys. Rep., 203, 1

[2] Blay, P. et al., 2006, A\&A, 446, 1095

[3] Bonning, E.W. \& Falanga, M. 2005, A\&A, 436, L31

[4] Corbet, R.H.D., Markwardt, C.B. \& Tueller, J., 2007, ApJ, 655, 458

[5] Crampton, D., Hutchings, J.B., Cowley, A.P., 1985, ApJ, 299, 839

[6] De Luca, A., et al. 2006, Science, 313, 814

[7] den Hartog et al. 2006, A\&A, 451, 587

[8] Farrell, S.A. et al., 2008, MNRAS, 389, 608

[9] Finger, M. H. et al, 2010, ApJ, 709, 1249

[10] Finley, J.P., Belloni, T., Cassinelli, J.P., 1992, A\&A, 262, L25

[11] Hall, T.A. et al. 2000, ApJ, 536, 450

[12] Haberl, F., White, N. E. 1990, ApJ, 361, 225

[13] Ikhsanov, N.R. 2007, MNRAS, 375, 698

[14] Kuiper, L. et al. 2006, ApJ, 645, 556

[15] Li, X.D. \& van den Heuvel, E.P.J., 1999, ApJ, 513, L45

[16] Mattana, F. et al. 2006, A\&A, 460, L1

[17] Postnov, K. et al. 2010, PoS, 015

[18] Reig, P. et al. 1996, A\&A, 311, 879

[19] Reig, P. et al., 2009, A\&A, 494, 1073

[20] Thompson, T. W. J. et al., 2007, ApJ, 661, 447

[21] Wang, W. 2008, edt. by Y. F. Yuan, X. D. Li \& D. Lai, AIP Conf. Proceedings, 968, 101

[22] Wang, W. 2009, MNRAS, 398, 1428

[23] Wang, W. 2010, A\&A, 520, 22

[24] Wang, W. 2011a, MNRAS, 413, 1083

[25] Wang, W. \& Li, J., 2011b, ApJ, submitted 